Voix et Images

volxetimages

\title{
" Mésaventure » : engendrement du poème et mythographie
}

\section{Liana Nissim}

Volume 9, numéro 2, hiver 1984

Roland Giguère

URI : https://id.erudit.org/iderudit/200439ar

DOI : https://doi.org/10.7202/200439ar

Aller au sommaire du numéro

\section{Éditeur(s)}

Université du Québec à Montréal

ISSN

0318-9201 (imprimé)

1705-933X (numérique)

Découvrir la revue

Citer cet article

Nissim, L. (1984). « Mésaventure » : engendrement du poème et mythographie.

Voix et Images, 9(2), 61-73. https://doi.org/10.7202/200439ar d'utilisation que vous pouvez consulter en ligne.

https://apropos.erudit.org/fr/usagers/politique-dutilisation/ 


\title{
"Mésaventure»: engendrement du poème et mythographie
}

\author{
par Liana Nissim, Université Catholique du Sacré-coeur, Milan, Italie
}

\section{Propos de lecture}

Forêt vierge folle ${ }^{1}$ le troisième recueil de Roland Giguère publié en 1978, a été accueilli par la critique avec un enthousiasme renouvelé pour ce peintre-poète; ${ }^{2}$ elle a vu dans ce livre le troisième volet de cette construction de l'imaginaire que Giguère lui-même a toujours déclaré être le but de chacune de ses créations, tant de parole que d'image. ${ }^{3}$

Toutefois il s'agit généralement de comptes rendus qui se proposent de présenter au lecteur l'ensemble du recueil; mais il n'y a pas encore de véritables essais centrés sur l'analyse détaillée des textes de ce dernier recueil.

Les éléments qui m'ont frappée, en tant que lectrice passionnée de Giguère, sont de deux ordres. D'une part, je suis moi-même convaincue que Giguère, dans la ligne de ses recueils précédents - l'Âge de la parole et la Main au feu 4 - a perpétué dans le respect de sa propre tradition, la structure mythographique sur laquelle se fonde son système d'interprétation du monde, le même qui avait été décelé, approfondi, systématisé par l'analyse

1. R. GIGUĖRE, Forêt vierge folle, Ed. de l'Hexagone, Montréal, 1978

2. Voir par exemple M. BEAULIEU, «Un poète magistral», le Livre d'ici, vol. 4, no 24 , 21.3.1979; R. MARTEL, «Roland Giguère artisan de la simple splendeur», La Presse, 24.2.1979; G. MARCOTTE, «Prendre tout pour une fenêtre», le Devoir, 3.2.1979; J. ROYER, «Pour célébrer Roland Giguère. La main libère la parole», le Devoir, 3.2.1979; R. MARTEL, «Pour nous donner la lumière», la Presse, 24.2.1979; M. LEMAIRE, «Multiple Giguère, Lettres québécoises, no 13, février 1979, pp. 17-19; F. HÉBERT, «Roland Giguère. Une poésie aux filets réversibles», Liberté, no 122, mars-avril 1979, pp. 124-127.

3. Giguère écrit, par exemple, dans «Au-delà» (Forêt vierge folle, p. 19): "La libération de l'homme par la poésie s'effectue: la triste réalité est bientôt remplacée par le rêve qui devient une seconde réalité, mais hors d'atteinte des circonstances extérieures qui pourraient la transformer's.

4. R. GIGUĖRE, l'Áge de la parole, Ed. de l'Hexagone, Montréal, 1965; la Main au feu, Ed. de l'Hexagone, Montréal, Montréal, 1973. 
très soignée de quelques critiques tels que Marcotte, Chamberland, Brochu, dont les essais sont fondamentaux pour la connaissance de Giguère. ${ }^{5}$ D'autre part j'ai cru pouvoir percevoir dans Forêt vierge folle un thème nouveau, qui n'apparaît, par ailleurs, que dans les poèmes les plus récents ${ }^{6}$ que je définirai pour le moment «contemplation du mythe» et que je chercherai à préciser dans mon analyse.

Dans cet article je me propose donc d'offrir ma modeste contribution à l'étude des procédés de production du texte qui ont constitué et qui constituent encore le caractère spécifique de la poésie giguérienne; mais en même temps je voudrais faire ressortir la nouvelle vision du poète par rapport à sa propre mythographie.

Pour ce type de travail j'ai choisi le poème «Mésaventure»:? il s'agit d'un texte très court et relativement simple, où le modèle de production textuelle propre à Giguère est à mon sens singulièrement exemplaire; en plus, c'est l'un des textes où la nouveauté à laquelle je faisais allusion plus haut constitue, me semble-t-il, l'authentique illustration:

\section{MÉSAVENTURE}

Dans un ciel de sang coule la mésange jusqu'au nid défait qui défend ses petits quand le tremble se couche à midi

le vent coupe comme une aile d'ange en ces temps de débris qui bat encore pour faire croire à la vie mais les amours durent malgré tout ce que j'écris sur vos vieux paravents

5. Les essais significatifs dédiés à Giguère étant très nombreux, je ne rappellerai ici que ceux qui me paraissent vraiment indispensables pour la connaissance de l'oeuvre du poète. G. MARCOTTE, «Roland Giguère», dans Une littérature qui se fait, HMH, Montréal, 1962, pp. 284-293; A. BROCHU, "Commentaire de 'Roses et Ronces'", la Barre du Jour, Déc.-Mai 1968, pp. 45-64; A. BROCHU, "La Main au feu», dans Livres et auteurs québécois 1973, Les Presses de l'Université de Laval, 1974, pp. 93-96; A. BROCHU, «Adorable femme des neiges», dans Dictionnaire des oeuvres littéraires du Québec, à paraître; P. CHAMBERLAND, «Lampe d'obsidienne», la Barre du Jour, cit., pp. 21-43; U. AYLWIN, "Introduction à l"âge' de Roland Giguère", la Barre du Jour, cit., pp. 11-17; C. BERTRAND, "Lecture de 'Yeux fixes'», la Barre du Jour, cit., pp. 84-92; F. GAGNON, «Le soleil noir, le piège et l'oiseau de malheur. Notes sur la symbolique graphique de Roland Giguère", la Barre du Jour, cit., pp. 111-112; M. VAN SCHENDEL, «Poésie et précision. 'Le défaut des ruines est d'avoir des habitants'», dans Présence de la critique, HMH, Montréal, 1966, pp. 195-196; R. GIGUÈRE, «D'un équilibre impondérable à une violence élémentaire, évolution thématique de la poésie québécoise 1935-1965», Voix et Images, VII, 1973, pp. 51-90; P. CHÂTILLON, «La naissance du feu dans la jeune poésie du Québec», dans Archives de lettres canadiennes, Fides, Montréal, 1969, pp. 255-282.

6. Comme pour l'Âge de la parole et la Main au feu, aussi dans Forêt vierge folle les premiers textes sont datés 1949: il s'agit encore une fois d'un itinéraire poétique complet, qui part de ses origines. Les derniers textes du recueil sont datés de 1977.

7. Forêt vierge folle, p. 209. 


\section{Premier niveau de lecture: la production du texte}

\subsection{Première strophe: l'apparente mimésis du réel}

Dès le titre «Mésaventure», le lecteur s'aperçoit qu'il est en présence d'un thème fondamental de la poésie de Giguère. L'événement catastrophique, le malheur qui frappe d'une façon inattendue, le bouleversement violent de la nature, la mésaventure: ce sont toutes des variantes (plus ou moins hyperbolisées) qui actualisent le même invariant structural désastre, code qui, chez Giguère, est toujours choisi pour la narration et la description de la condition humaine et universelle. ${ }^{8}$

Les deux premières strophes donc ne font que remplir l'attente du lecteur: elles sont l'expansion engendrée par le titre lui-même.

Ainsi la première image, qui introduit le lecteur dans le vif de la mésaventure, dans un ciel de sang, est directement générée par le titre; en effet, le stétéotype neutre, propre de la communication quotidienne, qui désigne le coucher du soleil, ciel rouge, est remplacé par son hyperbolisation métaphorique ciel de sang, qui contient dans son sème toute la négativité du sang répandu, signe de grave malheur et de mort violente. À cela, il faut ajouter que l'image est supportée par calque du cliché mer de sang: ${ }^{9}$ cette surdétermination signifie bouleversement de l'univers, selon les termes de l'opposition bas/haut - mer/ciel. Voilà le schéma sur la base duquel l'image a été engendrée:

haut

bas

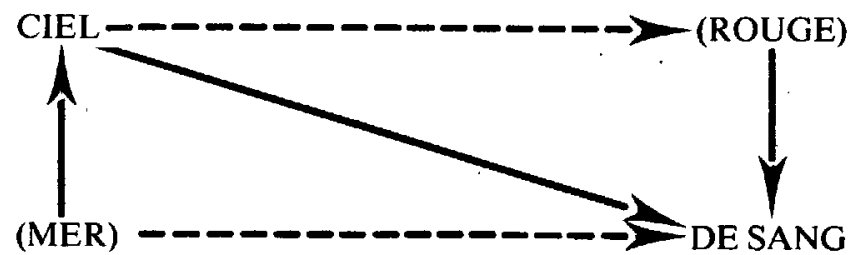

La séquence lexicale suivante, coule la mésange est issue à son tour des potentialités conjuguées du titre et de la première image.

$\grave{A}$ une première lecture, le verbe couler donne l'impression d'une mimésis convaincante de la soudaine descente en piqué d'un oiseau; en réalité, le choix de ce verbe est déterminé par deux éléments de la séquence précédente. D'un côté - sous l'impulsion de ce sang qui clôt la première séquence - coule est suggéré par le stéréotype le sang coule; de l'autre côté, couler est sémantiquement lié à mer qui (comme nous venons de le voir) est

8. «Le présent giguérien /.../ est le temps de la destruction, des émoussements, des pulvérisations, du retour à l'informe. Rien ni personne n'y échappe. Tout vire aux ruines et cendres..." écrit par exemple A. BROCHU dans son essai "La Main au feu», cit., p. 95.

9. Le cliché est employé par Giguère lui-même, par exemple dans «Yeux fixes ( $L$ 'Âge de la parole, p. 96) où il écrit marais de sang; ou dans "Les couleurs de l'Oracle» (la Main au feu, p. 94) avec marée de sang. 
refoulée dans le texte mais potentiellement présente dans ciel de sang. En effet, couler signifie sombrer, couler à pic, et il est ordinairement employé dans le rapport mer/navire. 10 Or, il se trouve que le mouvement impliqué dans le verbe couler (mouvement allant du haut vers ie bas) est effectivement réalisé par la mésange; mais le sujet du mouvement est un oiseau et ce mouvement se produit dans le ciel, pas dans l'eau; de plus, il va dans le sens contraire par rapport au mouvement de la première image, auquel nous avons fait allusion: voilà un autre signe de bouleversement de l'univers.

Venons-en maintenant au choix du mot mésange. Pourquoi un oiseau, et pourquoi cet oiseau?

Je crois qu'il est utile de rappeler que les oiseaux tiennent un espace important dans l'univers mythographique de Giguère: on les rencontre fréquemment dans les trois recueils; en outre ils appartiennent à plusieurs espèces.

Collaborateurs ou ennemis de l'homme, symboles de la détresse universelle ou signe magique et mystérieux d'un possibile rachat, ils constituent souvent une véritable obsession autant pour le poètell que pour le peintre - qui cherche sans répit à les reproduire dans ses dessins 12 - et ils témoignent de la profonde nécessité qu'il ressent de nommer les objets de son mythe. 13

Je citerai comme preuve (une parmi les nombreuses possibles, mais à mon sens très significative) la page 38 de Forêt vierge folle.

Quatre oiseaux y sont dessinés, dont le premier, qui envahit la page du haut, est un gros rapace; il menace un oiseau plus petit qui à son tour retient, prisonnier dans ses griffes, un oiseau minuscule. Un quatrième, à côté, replie ses ailes, comme s'il était blessé. Sous le dessin, Giguère écrit:

10. Le verbe est employé en ce sens par Giguère lui-même, par exemple dans «Pour tant de jours» (l'Áge de la parole, p. 15): "tant de radeaux coulés»; ou dans "La main de l'homme» (la Main au feu, p. 43): «il se jette à l'eau /.../ il coule dans le fond"; de la même manière il emploie couler à pic: «L'étranger enjamba la ligne d'horizon et coula à pic» (dans «Miror», la Main au feu, p. 27).

11. Pour la curiosité du lecteur: dans les trois recueils, les noms d'oiseaux apparaissent 73 fois.

12. Giguère écrit dans Forêt vierge folle, aux pp. 180-181: «Mais comment ècrire un oiseau? Comment peindre un oiseau? On prend sa plume, on choisit sa couleur et déjà il n'est plus tà, perdu dans son vol. Ne reste que son chant pour le musicien à l'écoute.

Pauvre oiseau, sans ailes, bec perdu, mal fait, sans même de griffes, où iras-tu ainsi, sinon dans un musée?»

13. «J'écrivais pour nommer, appeler, exorciser, ouvrir, mais appeler surtout» (Forêt vierge folle, p. 110) 


\section{LES OISEAUX NE PARLENT PAS \\ LES UNS CHANTENT \\ LES AUTRES DÉVORENT}

et il se trouve que les dévorés sont toujours

ceux qui chantent

Les dévorants ont pour noms

\section{L'AIGLE \\ LE VAUTOUR \\ LE CONDOR DES ANDES ET D'AILLEURS \\ (L'HOMME N'A NI BEC NI GRIFFES \\ IL PARLE ÉCRIT MARCHE ET FAIT LA GUERRE)}

Revenons à notre poème et au choix de la mésange. Le petit oiseau èst particulièrement bien choisi pour être victime d'une mésaventure: il appartient à la catégorie de ceux qui chantent, donc de ceux qui sont toujours menacés et sans défense. Mais cette fois encore le choix du mot mésange est avant tout un choix linguistique. Parmi les nombreux oiseaux frêles et chanteurs (qui sont effectivement présents dans d'autres poèmes) Giguère choisit à dessin la mésange (et ici seulement: dans toute son oeuvre le mot n'a jamais été utilisé) parce qu'elle participe du titre, lequel - il ne faut pas l'oublier - est le noyaux sémantique qui provoque l'engendrement de ce texte - ; et en même temps mésange contient le mot ange du vers 4:

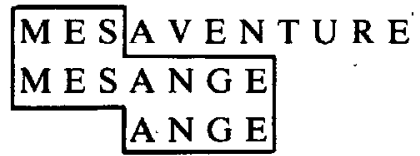

Le titre et ce premier vers dont je viens de proposer une analyse sémantique sont donc étroitement liés et se justifient pour des raisons formelles (les seules, en réalité, qui comptent dans la définition de la littérarité d'un texte); ce qui est prouvé aussi par les importantes récurrences phonétiques, qui n'auront sans doute pas échappé au lecteur. En effet le son $/ a /$, martelant et continu, revient quatre fois de suite:

\section{MesavENture}

DANs un ciel de $s A N g$ coủle la mésANge

D'ailleurs le même son $/ \widetilde{a} /$, si massivement présent en ouverture, revient continuellement en écho au cours des deux premières strophes:

v. 2 défENd

v. 3 quANd trEMble

v. 4 vENt ANge

v. 6 EN tEMps ENcore

À la troisième strophe, le son / $\widetilde{a} /$ semble disparaître (nous verrons d'ailleurs qu'il y a un décalage profond entre les deux premières strophes et la dernière). Mais le voilà, de nouveau, dans le mot conclusif, véritable écho du titre et du premier mot de la deuxième strophe qui confirme le pouvoir d'engendrement du titre «Mésaventure»: 


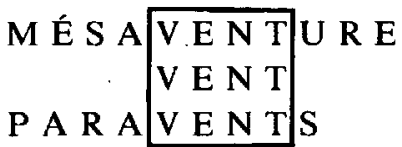

Mais revenons à la première strophe. Au v. 2 la dominante phonétique a changé: il s'agit maintenant de la voyelle /i/, elle aussi présente trois fois dans le vers (ainsi que /ă/ l'était dans le v. 1):

$$
\text { jusqu'au nId défait quI défend ses petIts }
$$

/i/ aussi revient (moins fréquemment toutefois) au cours du poème, et souvent en position forte:
v. 3 mIdI
v. 5 débrIs quI
v. 6 vIe
v. 8 j'écrIs

Cet aperçu de la structuration phonétique du texte est une preuve ultérieure du conditionnement formel qui existe à l'intérieur d'un poème à l'apparence si linéaire.

De même, du point de vue sémantique, le v. 2 aussi est fortement motivé par les séquences verbales qui le précèdent.

N'importe quel désastre frappe l'homme et métonymiquement ce qui le représente le mieux: sa maison. Ici cependant la narration du désastre est présentée selon le code «ornithologique»: à homme-maison Giguère préfère la métaphore oiseau-nid.

Le vers dans son ensemble est tout de suite accepté par le lecteur, parce qu'il représente un stéréotype littéraire: le nid qui défend les petits de la fureur de la tempête est une image qui revient souvent dans certaines poésies intimistes ou enfantines, dans des comptines et de petits contes moralisants. Il s'agit donc d'un matériel métaphorique préexistant au texte giguérien. Et c'est exactement sa banalité qui fait passer inaperçue l'anomalie de défait. Le lieu commun en effet donne une sensation de parfaite cohérence réaliste. Au contraire: comment un nid défait peut-il être une vraie défense pour les petits? S'il est défait, il n'est pas à même de défendre; mais le parallélisme phonétique défait / défend s'ajoutant au lieu commun, efface entièrement la contradiction du point de vue de la mimésis du réel; et ainsi défait peut rester dans le texte, pour signifier, lui-aussi, la situation de bouleversement.

Le v. 3, quand le tremble se couche à midi, complète l'expansion descriptive de la mésaventure qui a frappé la mésange: l'arbre qui abritait le nid, ébranlé à son tour et contraint de se ployer, clôt l'image, qu'on pourrait définir comme pyramidale, celle-ci allant du contenu au contenant, selon la séquence:

$$
\text { oiseau }
$$


Avec l'achèvement du tableau, l'effet-mimétique de la réalité apparaît encore plus accentué. Toutefois, des éléments de surdétermination formelle sont présents même dans ce vers; en particulier, le verbe se coucher employé pour décrire l'arbre incliné par la violence du vent, présente des relations formelles (tant phonétiques que sémantiques) avec les autres mots du texte. D'un point de vue phonétique, se couche propose le même groupe initial que les autres verbes choisis pour signifier le malheur; c'est donc le verbe coule du v. 1 qui est à l'origine de la séquence:

$$
\text { COUle } \longrightarrow \text { se COUche } \longrightarrow \text { COUpe }
$$

D'un point de vue sémantique, le verbe se couche recèle encore une fois le bouleversement que j'ai déjà eu l'occasion de mettre en évidence dans les deux vers précédents. Avant tout, ce sens de bouleversement est contenu dans le sème propre du mot; la définition donnée par le dictionnaire est en effet: «Rapprocher de l'horizontale (ce qui est naturellement vertical)». Il s'agit donc d'un mouvement qui se réalise dans un sens contraire à cèlui que la nature de l'objet exigerait. En outre, le verbe se coucher, dans le langage employé couramment pour décrire.la nature, indique aussi le mouvement du soleil qui descend et se cache sous l'horizon. Le mot matriciel «soleil» est refoulé dans le texte; toutefois, il ne faut pas oublier qu'il était déjà signifié dans ciel de sang du. v. 1.

Le ciel rouge de ce vers, comme ici le verbe se couche suggèrent l'heure de l'événement: l'heure du coucher. Or, le mot midi, présent dans le texte, est parfaitement justifié du point de vue de la mimésis du réel: il désigne le point cardinal vers lequel l'arbre se courbe. Mais midi est le mot par lequel on désigne aussi le milieu du jour, le moment où le soleil eśt à son comble dans le ciel. Le choix linguistique midi rapproché du verbe se couche présente donc quelque chose de bouleversant: on ne peut pas se passer de lire par transparence le soleil se couche à midi, surimpression au stéréotype dont on parlait plus haut, le soleil se couche à l'horizon. L'obscure sensation du lecteur de se trouver vis-à-vis d'un coucher de soleil qui se vérifie au moment où le soleil est à son comble, accentue, me semble-t-il, l'impression générale d'un bouleversement de la nature.

\subsection{Deuxième strophe: l'irruption du surréel}

Jusqu'ici (on vient de le voir) le poème obéissait scrupuleusement aux lois de la mimésis du réel. Tout change cependant dans la deuxième strophe, où Giguère (confirmant sa nature de poète fondamentalement surréaliste) enfreint incontestablement les règles de la vraisemblance, en permettant au lecteur de saisir de façon plus immédiate l'engendrement linguistique du texte, au-delà et en dehors de toute préoccupation de cohérence «réaliste». Et c'est effectivement dans cette strophe qu'on retrouve des structures thématiques qui sont exemplaires de la vision du monde de Giguère. 
Le v. 4 s'ouvre avec le vent: ce mot fonctionne comme lien avec la description apparemment réaliste de la première strophe. Nous savons déjà, en effet, que le tremble est courbé, couché plutôt, à midi; la cause première de la mésaventure qui frappe le petit oiseau et son nid ne peut donc être que le vent. Toutefois, vent (comme plus haut mésange) est directement engendré par le titre; et ici il ne s'agit pas tellement d'une production sémantique, mais plutôt d'une production lexicale; en effet - on l'a déjà vu - le mot vent est graphiquement contenu dans mésaventure, ainsi que la première partie de mésange:
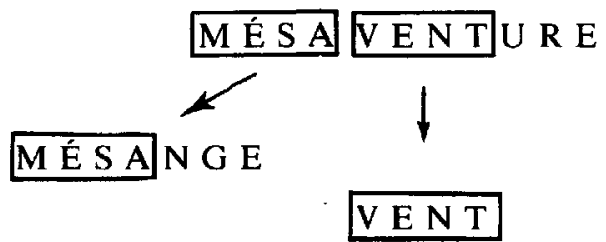

Le vent produit à son tour la séquence subséquente; mais c'est à ce moment-là que le mécanisme d'apparente fidélité à la vraisemblance s'efface, pour céder la place à une structure typiquement surréaliste.

La structure grammaticale le vent coupe comme est parfaitement acceptable pour le lecteur; mais le mot aile survient tout à fait à l'improviste. On s'attendrait par exemple à couteau, lame; mais quel rapport peut-il y avoir entre l'air qui coupe le visage et une aile? Du point de vue de la vraisemblance aucun rapport n'est admissible: une aile ne coupe pas. Ici, il ne s'agit donc plus d'un phénomène de polysémie du mot ou de culmination sémantique, comme c'était le cas dans la strophe précédente, mais c'est au contraire la superposition et la contamination de deux systèmes cohérents, qui donnent le choc de quelque chose d'absolument inattendu:

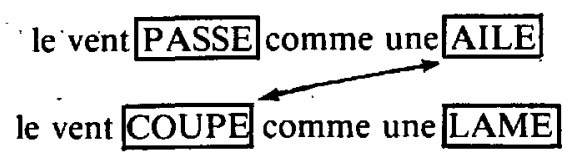

Cependant, la séquence une aile d'ange n'est pas indépendante du texte, car d'une part le passage du vent (on vient de le voir) peut très bien faire penser à l'effet d'une aile gigantesque; d'autre part le lecteur perçoit le lien sémantique, que j'ai déjà souligné, entre mésange et ange; enfin on ne peut pas oublier que la protagoniste, la mésange, est pourvue d'ailes. Cette connexion des mots - avec son apparence d'écriture automatique - donne lieu à la naissance d'une image imprévue mais puissante: l'image d'un ange qui domine la terre de son aile immense, en y répandant ruine et mort: l'image de l'ange exterminateur. Nous en avons la confirmation tout de suite après dans la séquence en ces temps de débris. 
Ces quelques mots renferment l'un des lieux les plus caractéristiques de la mythographie giguérienne. Pour mieux le comprendre, il faut revenir un instant sur ce que j'ai défini comme un invariant structural très fréquent dans la poésie de Giguère: le thème du désastre. À l'intérieur du code désastre (et je l'appelle code puisque le système désastre ne se développe jamais en soi, mais il est toujours employé comme langage hyperbolique pour représenter l'anthropologie giguérienne), deux paradigmes de variantes peuvent être engendrés, avec une différente structuration temporelle.

Un premier paradigme est représenté par la description du désastre qui est en train de se produire. Il s'agit par exemple d'un volcan qui crache mille soleils, ou d'un ciel déchiré de cris, ou du feu qui brûle tout, du vent qui vient abattre les totems, du soleil qui se dédouble pour entrer dans les yeux d'un navire en détresse... les variantes sont infinies. Dans notre cas (j'ai déjà souligné qu'il s'agit ici d'une variante atténuée) c'est la mésaventure qui, dans un ciel de sang, frappe la petite mésange, son nid, son arbre.

Souvent toutefois la description du désastre qui est en train d'arriver, se greffe (d'une façon ordinairement mystérieuse, inexpliquée) sur un autre désastre, qui s'est déjà produit, on ne sait pas quand ni comment: et c'est le deuxième paradigme dont je parlais.

Ce type de désastre signifie toujours chez Giguère la désolation générale qui pénètre tout, les hommes et les choses, et qui concerne le passé le plus récent ou, plus souvent encore, le temps présent. Feux morts, visages glacés, pays désertés, forêts muettes et décapitées, cendres et décombres, objets brisés, nuages de sang, mares de boue, déserts envahissants: tout un monde à moitié détruit et agonisant, sans espoir et sans vie, qui aurait survécu après une énorme catastrophe universelle.

Dans «Mésaventure», la même représentation mythographique se répète: la mésaventure de la mésange arrive dans un monde qui est déjà irrémédiablement détruit. Cependant, cette fois aussi le ton a été atténué: les images souvent violentes qui se succèdent dans tant de textes giguériens pour représenter le désastre qui a frappé le monde, sont ici brièvement résumées dans la séquence en ces temps de débris et dans l'expansion successive: l'aile de l'ange bat encore pour faire croire à la vie.

En effet, c'est dans cette illusion de vie (espérance mensongère pour les larves survécues dans le monde postapocalyptique qu'est l'époque présente) qu'un événement nouveau est possible. Dans la débâcle universelle quelque chose d'individuel peut encore arriver: tantôt c'est un autre désastre qui se produit, tantôt c'est le commencement d'un long et difficile chemin de salut.

Récapitulons cette première partie de notre analyse. Nous sommes en présence de la description de deux désastres, comme il arrive dans beaucoup d'autres textes de Giguère: l'un, plus général, est la condition universelle 
(ces temps de débris) sur laquelle se greffe le désastre individuel de la mésange.

Cet événement est présenté sur un ton mineur, plus bref, plus sec qu'il n'arrive habituellement dans la poésie de Giguère: seulement.quelques traits rapides, à peine ébauchés, comme pour évoquer quelque chose qu'on connaît déjà.

C'est peut-être grâce à cette extrême simplicité d'écriture que nous avons pu parcourir le texte mot à mot: nous avons ainsi analysé les moyens de production textuelle qui caractérisent l'écriture de Giguère; ces moyens sont liés aux valeurs phonétiques (alternances, récurrences) aussi bien qu'aux valeurs sémantiques (surdétermination par calque et analogie de mots-valises), pour une écriture qui, dans la première partie, paraît parfaitement fidèle à la mimésis du réel, tandis que, dans la deuxième partie, elle revient à la manière surréaliste de Giguère.

\section{Deuxième niveau de lecture: la contemplation du mythe}

La troisième strophe (qui va nous obliger à une lecture différente du poème) s'ouvre par le vers: mais les amours durent, qui ne fait que compléter le mythe giguérien. En effet, dans ses poèmes, l'espoir ne meurt jamais entièrement: il y a toujours quelque chose - un petit signe, un objettalisman, un sentiment craintif et délicat - qui survit dans le désastre et qui souvent se constitue comme point de départ pour un chemin nouveau. $\dot{A}$ l'invariant désastre donc, s'oppose l'invariant de quelque chose qui dure, et ce quelque chose est souvent actualisé par la variante de l'amour, seule lumière dans la nuit, silencieuse et calme attente que le désastre prenne fin, exaltante redécouverte de l'espoir et du besoin de survivre et de vivre.

La structuration du mythe est entièrement présente dans «Mésaventure»: nous avons vu le désastre qui est en train de se produire, se greffant sur les débris qui seuls restent désormais à signifier le temps présent, qui est mort et ne peut que simuler la vie. Maintenant nous lisons: mais les amours durent, où l'adversative initiale mais ouvre l'espoir qui avait été nié avec tant d'acharnement dans les deux strophes précédentes.

Toutefois, si d'un point de vue du référent ce vers constitue un renversement de la situation, d'un point de vue formel il est, comme les autres, un déroulement des potentiels du mot initial, mésaventure.

En effet, il contient graphiquement la dernière partie du mot du titre, comme à conclusion d'un cycle:

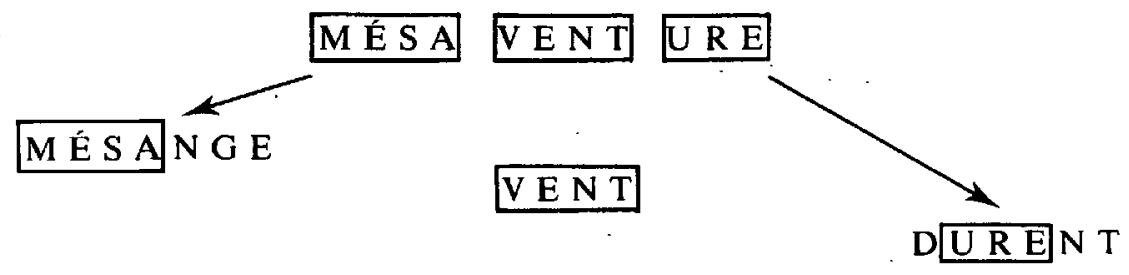


Toutefois, dans ce cas aussi, nous sommes en présence d'un style bien changé par rapport à celui qui est propre à Giguère. Aucune narration n'est faite ici de l'aventure salutaire de l'amour, comme il arrivait au contraire dans tant d'autres textes. ${ }^{14} \mathrm{De}$ la même manière que, pour les deux premiêres strophes, tout se réduit à très peu de mots, comme si le poète voulait rapidement rappeler, seulement ébaucher la dernière composante de sa vision du monde.

C'est dans les derniers' vers que nous trouvons l'explication de cette nouvelle écriture et, en même temps, un sens nouveau aux vers que nous avons lus jusqu'ici.

Après l'assertion mais les amours durent, on s'attendrait à une concessive du type: «malgré les désastres et les mésaventures qui frappent le monde». Rien de semblable, au contraire: sans aucun lien apparent avec le texte qui les précèdent, les deux derniers vers apparaissent, absolument inattendus et obscurs:

malgré tout ce que j'écris

sur vos vieux paravents

À première vue, le lecteur est totalement déconcerté: son attente a été déçue, contrairement à ce qui se passait dans les vers précédents; des éléments incompréhensibles suggèrent la présence d'une signification inédite.

Qu'est-ce que vient faire ici l'acte d'écriture? Et le poète, qu'est-ce qu'il écrit sur les vieux paravents? Je pense que, pour le comprendre, il faut faire une comparaison entre le texte hypothétique auquel le lecteur s'attendait, et le texte effectivement réalisé.

On s'attendait à lire

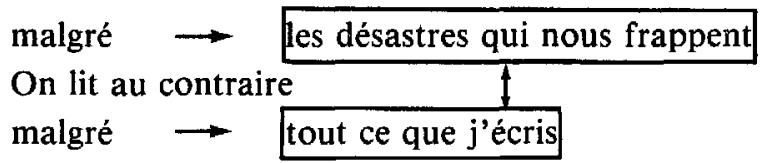

Le poète, à l'intérieur d'une même structure grammaticale, a effectué une substitution de référent: l'acte d'écriture (tout ce que j'écris) se substitue à la réalité du désastre. Le désastre n'existe donc plus, sinon comme objet de l'écriture, comme évocation. Giguère ne vit plus de son mythe, dans son mythe: il s'en souvient, il l'écrit. Et il l'écrit sur de vieux paravents. Ce mot, paravents, nous force à une longue réflexion, à cause de l'étonnante polysémie du dernier vers.

14. Je rappellerai, pour donner au moins un exemple, le poème de 1951, «À l'ombre de ma vie" (Forêt vierge folle, pp. 29-34) où le mythe entier - du désastre au salut par l'amour - est raconté en 160 vers. 
Bien sûr, paravents est engendré par le vent de la deưxième strophe, et à dessein il se pose comme son opposition: paravent est, dans son sens étymologique, quelque chose qui est destiné à protéger contre le vent, ce vent qui (on s'en souvient) se déchaînait sur une nature bouleversée; mais le paravent est un fragile objèt d'intérieur, et en plus il s'agit ici de vieux paravents, ce qui rend le meuble encore plus délicat, inutile, décoratif.

Voilà donc que toute la mésaventure des deux premières strophes est réduite à une dimension presque risible: il suffit d'un vieux paravent pour que le vent furieux qui frappait l'arbre et le nid soit neutralisé.

Et encore: le paravent n'est sûrement pas l'objet d'un intérieur moderne; il rappelle plutôt une certaine atmosphère du dix-septième siècle, à la fois sereine et insouciante. Vieux souligne davantage cette atmosphère du temps passé, quand des rendez-vous galants étaient donnés derrière de jolis paravents.

Nous comprenons alors ce pluriel, les amours, qui enlève tout caractère dramatique mais en même temps diminue la force extraordinaire de l'amour. Ceci est encore souligné par vos, qui accompagne les vieux paravents: la femme, maîtresse des paravents, est vouvoyée, comme une ancienne dame, comme dans une aventure galante du passé libertin.

Cette atmosphère nouvelle, si étrangère au mythe giguérien et au texte même de «Mésaventure», nous force à une révision de notre lecture initiale qui suivait la progression des séquences verbales, et, en lecture rétroactive, nous permet de découvrir la signification plus profonde du texte.

J'écris est employé dans un sens ambivalent: d'une part le poète écrit effectivement (nous sommes en présence d'un poème, d'un texte écrit). D'autre part, peut-être, il dessine, il peint: sur de vieux paravents il est plus logique de peindre que d'écrire; ceci prouve que pour Giguère en réalité écrire et dessiner s'identifient. 15

Mais, qu'est-ce qu'il écrit/dessine, le poète-peintre, sur les vieux paravents?

À la manière de certains miniaturistes japonais, il trace quelques sveltes lignes stylisées: un ciel rouge, un oiseau qui descend en piqué, un arbre courbé par le vent, et l'aile d'un ange peut-être. Rien de plus habituel pour les dessins qui décorent les paravents anciens, sauf peut-être le dernier détail, l'aile d'un ange qui domine de mystérieux débris. Cette image est plutôt un écho de certains poèmes de Mallarmé: le mystère de l'Absolu traduit dans le silencieux balancement d'un plumage qui disparaît ou bien les paradis farouches qui surgissent du'vol d'un éventail (étrange analogie d'atmosphère entre cet éventail sur lequel en encre rouge Mallarmé écrivait l'histoire d'une quête métaphysique et les vieux paravents sur lesquels Giguère écrit l'histoire du destin universel!) 
Mais revenons à la lecture rétroactive dụ texte et à la conclusion de notre analyse: la préoccupation de vraisemblance réaliste qui paraissait inspirer la prèmière strophe était parfaitement illusoire. Il ne s'agit pas ici de mimésis du réel, mais au contraire d'une mémisis qui traduit soigneusement en parole un joli dessin.

Dans ce dessin, par des traits rapides et symboliques, Giguère rappelle son ancien mythe. Il ne l'a donc pas oublié, sa vision du monde n'a pas changé. Mais desormais le mythe ne représente plus la vie du poète; il l'aime toujours, mais il en est assez loin. Sa vie, sa préoccupation existentielle maintenant, est celle d'écrire, de desssiner. La recherche d'écriture a transformé la vitalité du mythe dans la contemplation de celui-ci.

Et si l'aile qui bat sur des débris est effectivement l'image choisie pour représenter la phase la plus dramatique du mythe, ce qui suit, pour faire croire à la vie, nous apparaît maintenant bien plus amphibologique qu'on avait cru pendant notre première lecture: l'aile qui bat pour donner une illusion de vie, mais qui en réalité est dessinée sur les paravents, est peut-être une allusion à l'apparence de la vie, à la création de la vie qui depuis des siècles constitue le désir le plus profond de chaque artiste.

Et alors, l'événement nouveau, le chemin vers le salut, la vie, ne serait rien d'autre que l'acte d'écriture.

Aucune action n'est possible en ces temps de débris, sauf celle de faire croire à la vie en la représentant par l'art.

15. Giguère écrit dans Forêt vierge folle, p. 111: «De l'âge de la parole je passais à l'âge de l'image et pour moi il n'y avait là nulle abdication, nulle rupture; je disais - et je dis encore - les mêmes choses, autrement. Cet univers qui sur la feuille maintenant devenait visible était bien le même qui hantait mes poèmes. Et je vais toujours ainsi du poème au dessin, à la peinture; du mot à la ligne, à la couleur, pour dire et pour voir et pour donner $a$ voir». 


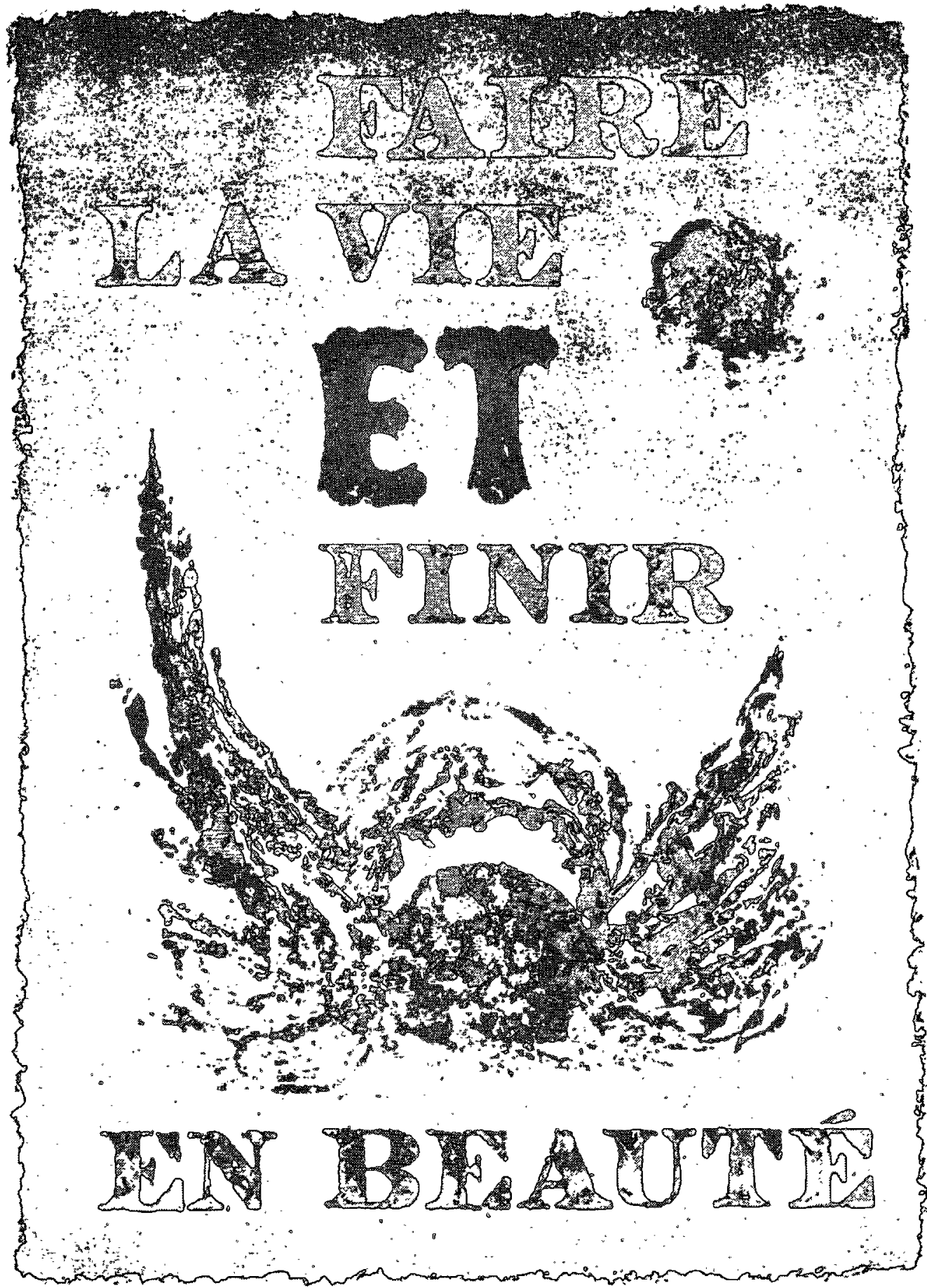

Roland Giguère, sans date. 